\section{e0479 IMPACTS OF SUCCESSFUL PERCUTANEOUS CORONARY INTERVENTION FOR CHRONIC TOTAL OCCLUSIONS ON PATIENTS WITH HEART DYSFUNCTION}

doi:10.1136/hrt.2010.208967.479

Yaling Han, Xianhua Yi, Shouli Wang, Quanmin Jing. Shenyang Northern Hospital

Introduction Successful percutaneous coronary intervention (PCI) for chronic total occlusion (CTO) had proven benefit for long-term survival. However, its impact on heart function of patients with heart dysfunction is uncertain. The purpose of the present study was to analyse the impacts on heart function of patients with heart dysfunction who underwent successful PCI for CTOs.

Methods Between June 1993 and December 2007, the clinical data of 472 consecutive patients with heart dysfunction in our center underwent PCI for CTOs were analysed. The mean age was $59.4 \pm 11.5$ years. These patients were divided into two groups according to the procedural success $(n=421)$ or failure $(n=51)$, in order to compare the heart function between groups. A follow-up echocardiogram examination was performed 6 months after PCI.

Results 391 (92.9\%) patients in CTO success group and 46 (90.2\%) patients in CTO failure group accepted the examination of echocardiogram at mean time of $6.2 \pm 1.7$ months. For patients underwent success CTO revascularisation, left ventricular ejection fraction (LVEF) was increased from $44.5 \pm 3.2 \%$ to $50.2 \pm 5.7 \%(p<0.05)$, and left ventricular end-diastolic volume index (LVEDVI) was declined from $84.7 \pm 14.3 \mathrm{ml} / \mathrm{m}^{2}$ to $78.4 \pm 13.7 \mathrm{ml} / \mathrm{m}^{2}$ ( $\left.\mathrm{p}<0.05\right)$. But the LVEF and LVEDVI had no significant changes in the CTO failure group $\left(42.5 \pm 4.0 \%\right.$ vs $43.6 \pm 4.1 \%$ and $86.8 \pm 14.4 \mathrm{ml} / \mathrm{m}^{2}$ vs $85.9 \pm 14.7 \mathrm{ml} /$ $\mathrm{m}^{2}$, both $\left.\mathrm{p}>0.05\right)$, respectively. In addition, stage of NYHA classification was improved in most people in CTO success group $(p<0.05)$. Conclusions Successful procedures of CTO leads to the improvement of left ventricular function of patient with heart dysfunction.

\section{e0480 LONGTERM CLINICAL OUTCOME OF PATIENTS UNDERGOING SUCCESSFUL PERCUTANEOUS CORONARY INTERVENTION FOR CHRONIC TOTAL CORONARY ARTERY OCCLUSION}

doi:10.1136/hrt.2010.208967.480

Yaling Han, Xianhua Yi, Shouli Wang, Quanmin Jing, Yingyan Ma, Xiaozeng Wang. Shenyang Northern Hospital

Introduction There are limited data on the impact of successful chronic total occlusion (CTO) revascularisation by percutaneous coronary intervention (PCI) on long-term outcomes.

Methods Between June 1993 and December 2006, a total of 1332 patients having PCI for a CTO were consecutively registered. In addition to an assessment of procedural outcomes, patients were followed long-term for occurrence of major adverse cardiac events (MACE). A CTO was defined as an occlusion of the artery present for at least 3 months with Thrombolysis in Myocardial Infarction flow grade 0 or 1. Long-term survival rate was estimated by Kaplan-Meier methods. Results The overall success rate was $90.2 \%$ (1202/1332). During a median follow-up of $6.26 \pm 2.52$ years (median 6 years), the estimated 10 -year survival rate was $76.7 \%$ in the CTO success patients and $65.5 \%$ in the CTO failure patients, respectively $(p<0.001)$. The MACE-free survival rate was significantly higher for CTO success patients compared with CTO failure patients ( $48.0 \%$ vs. $33.0 \%$, p $<0.001)$. The rates of coronary artery bypass (CABG) were $4.3 \%$ vs. $14.6 \%(p<0.001)$ for the CTO success and CTO failure patients, respectively. Multivariate analysis showed that procedural failure, diabetes mellitus and multivessel disease were independent predictors of death.

Conclusions Successful revascularisation for CTO improves longterm survival and reduces MACE rate and the need for CABG compared with failed procedure.

\section{e0481 EFFICACY AND SAFETY OF TIROFIBAN TREATMENT COMBINED WITH PERCUTANEOUS CORONARY INTERVENTION IN THE ELDERLY WITH ACUTE ST SEGMENT ELEVATION MYOCARDIAL INFARCTION}

doi:10.1136/hrt.2010.208967.481

Huang Wei Guang, Wu Tong Guo, Luo Jing Yun. Department of Cardiology Guangzhou Red Cross Hospital

Objective To analyse the efficacy and safety of tirofiban treatment combined with percutaneous coronary intervention (PCI) in the elderly with acute ST segment elevation myocardial infarction prospectively.

Methods From May 2007 to May 2009, 104 patients who presented with acute STEMI within $12 \mathrm{~h}$ from onset and received successful primary PCI were enrolled into the study. All patients had angiographic evidence of initial total occlusion of infarct-related artery and finally restored toTIMI3 flow after PCI - All the patients were grouped into the tirofiban group $(\mathrm{n}=54)$ and the control group $(n=50)$ according to whether tirofiban was used or not Assessment of myocardial perfusion included Myocardial Blush Grades (MBG), and the resolution of the sum of ST-segment elevation (sumSTR) at $90 \mathrm{~min}$ after the procedure. Left ventricular ejection fraction (EF) was measured one week later. Major adverse cardiac events in hospital and bleeding complications were also assessed.

Results Baseline clinical and angiographic characteristics of the two groups were similar. Significant higher rates of MBG 3 were observed in the tirofiban group $(88.9 \%$ vs $58.0 \%, \mathrm{p}<0.05) \cdot$ Patients received tirofiban were more likely to achieve higher sumSTR $(70.3 \%$ vs $42.0 \%, \mathrm{p}<0.05)$. Ejection fraction was also increased markedly in tirofiban group than the control group $(56.2 \pm 7.6$ vs $46.7 \pm 8.5, \mathrm{p}<0.05) \cdot$ In-hospital major adverse cardiac events were not different between the two groups. There were slightly more minor bleeding complications in the tirofiban group compared with the control $(11.1 \%$ vs $6 \cdot 0 \%, \mathrm{p}>0.05) \cdot$ No patient had major bleeding or thrombocytopaenia.

Conclusion Tirofiban can further ameliorate microvascular perfusion and is safe and feasible for patients with STEMI undergoing primary PCI.

e0482 THE EFFICIENCY AND SAFETY OF THE SEEK ASPIRATION THROMBECTOMY CATHETER AND TIROFIBAN IN PRIMARY PERCUTANEOUS CORONARY INTERVENTION OF ACUTE MYOCARDIAL INFARCTION

doi:10.1136/hrt.2010.208967.482

Huang Wei Guang, Wu Tong Guo, Luo Jing Yun, Zao Qiang. Department of Cardiology Guangzhou Red Cross Hospital

Objective To assess the effect of the Zeek aspiration thrombectomy catheter and tirofiban in the myocardial reperfusion and clinical prognosis in patients with acute ST-segment elevation myocardial infarction (AMI) who were undergone primary percutaneous coronary intervention (PCI).

Methods 84 patients of AMI with thrombotic burden lesion confirmed by coronary angiography were randomly divided into the aspiration catheter and tirofiban group $(\mathrm{n}=42)$ and standard percutaneous coronary intervention $(\mathrm{PCI})$ group $(\mathrm{n}=42)$. Compared the TIMI flow grade, myocardial blush grade (MBG), ST segment resolution, left ventricular ejection fraction (LVEF) post PCI, haemorrahage and major adverse cardiac events (MACE) rate during hospitalisation between two groups.

Results Compared with control group, the study group improved TIMI flow grade, improved MBG and high LVEF, while the difference of MACE and haemorrahage rate during hospitalisation was nonsignificant. 
Conclusion In AMI patients treated with primary PCI, Combination of thrombus aspiration and tirofiban was safe and effective, which could lower thrombosis burden, improve distal myocardium perfusion and cardiac function after procedure, did not increase the incidence of MACEs.

\section{e0483 THE CLINICAL RESEARCH FOR ENDOVASCULAR TREATMENT OF DEBAKEY III AORTIC DISSECTION RECEIVED DOMESTIC THIN STEEL BINDING STENTS GRAFTING}

doi:10.1136/hrt.2010.208967.483

Liao Wei, Zhou Aiqin. The Affiliated Hospital of Gannan Medical College

Objective To evaluate the efficacy and safety of endovascular treatment for Debakey III aortic dissection by domestic thin steel Binding stents grafting.

Methods Between October 2006 to March 2010, 42 patients (all male, average age $(53.5 \pm 12.8)$ years, range $43 \sim 70$ years old) with Debakey III aortic dissection was treated with domestic thin steel Binding stents grafting, used an innovative transmission and delivery methods in all patients, of which branch stent were inplanted when the distance from the break of descending aorta to left subclavian artery is less than $10 \mathrm{~mm}$.

Results 42 patients were successfully implanted 48 thin steel Binding stents, including four branch stents. After the operation, six patients were verified endoleak, 3 of the patients were resolved by repeated stent distension and 3 cases were treated by placement of another stent. Three months later, one patient showed new rupture at the remote port of the stent and then was successfully implanted a new stent. Four cases showed numbness of right lower extremity and 1 case showed intermittent claudication of it. In follow-up of 3-32 months, MRI or CT showed the reduce of the false cavity with the formation of intraluminal thrombus, the enlargement of true cavity, and no complications such as tumour rupture, internal leakage and stent displacement in all of the patients.

Conclusions Endovascular thoracic aorta repair, with domestic thin steel Binding stents grafting and the innovative methods of interventional therapy, is an effective, less invasive and safe surgery with faster postoperative recovery, higher success rate and fewer complications for patients with Debakey III type aortic dissection, especially applicable to high-risk patients.

\section{e0484 THE SAFETY STUDY OF TIROFIBAN IN PATIENTS WITH STEMI DURING EMERGENCY PCI}

doi:10.1136/hrt.2010.208967.484

Guo Lai-jing, Hu Dayi, Huo Yong, Tang Oiang, Zhang Huiying, Xing Liying, Wang Zhi, Zhang Shuhe, Shi Zhentao, Zhu Zhenzhong. Chronic Disease Institute, Beijing University Shougang Hospital

Objective To assess the safety of GP IIb/IIIa antagonists tirofiban in patients with ST elevation myocardial infarction (STEMI) during emergency Percutaneous Coronary Intervention (PCI).

Method 120 patients with STEMI during emergency PCI, divided into two groups, tirofiban+PCI group $(n=60)$ and primary PCI group $(n=60)$. The two groups are compared on their major adverse cardiovascular events (MACE) rates which consisted of death, new onset myocardial infarction, persistent myocardial ischeamic state, Cardiac function (1eft ventricular ejection fraction) after operation, compression haemostasia time and adverse drug effect while in hospital.

Result Major adverse cardiovascular events (MACE) rates in tirofiban + PCI group was higher than that of primary PCI group $(10 \%$ vs $6.7 \%$ ); Postoperative heart function was better in tirofiban $+\mathrm{PCI}$ group than that in primary PCI group (56.97 \pm 8.41 vs $54.15 \pm 7.11$ ): There was no difference in severe haemorrhage between the two groups. The bleeding event rates were of higher tendency in tirofiban+PCI group. ( $8.3 \%$ vs $3.3 \%$ ). APTT and compression haemostasia time of tirofiban + PCI group were significant when prolonged $(53.97 \pm 10.58$ vs $32.51 \pm 6.31) \mathrm{p}<0.05)$, but no more bleeding and heamatom events occurred during prolongation.

Conclusion GPII b/III a antagonists tirofiban+PCI is a possible safe and effective reperfusion method with STEMI during emergency PCI.

\section{e0485 CLINIC STUDY OF DOMESTIC TIROFIBAN ON TIMI FLOW DURING FACILITATED PCI}

doi:10.1136/hrt.2010.208967.485

${ }^{1}$ Guo Lai-jing, ${ }^{1} \mathrm{Hu}$ Dayi, ${ }^{1}$ Huo Yong, ${ }^{1}$ Tang Qiang, ${ }^{1}$ Zhang Huiying, ${ }^{1}$ Xing Liying, ${ }^{1}$ Wang Zhi, ${ }^{2}$ Ou Huaqing, ${ }^{1}$ Shi Zhentao, ${ }^{3}$ Zhu Zhenzhong, ${ }^{1}$ Yan Do, ${ }^{1}$ Tang Ounzhong, ${ }^{1}$ Chen Xuezhi, 'Liu Xiumin. ${ }^{1}$ Department of Cardiology Beking University Shougang Hospital; ${ }^{2}$ Department of Cardiology Beking University Shougang Hospital; ${ }^{3}$ Department of Emerergency Beking University Shougang Hospital

Objective To assess Thrombolysis In Myocardial Infarction (TIMI) flow of the nation-produced tirofiban in patients with ST-segment elevation myocardial infarction (STEMI) during primary Percutaneous Coronary Intervention (PCI).

Methods 120 patients with STEMI during primary PCI from Nov 2006 to June 2009 were divided into two groups, tirofiban $+\mathrm{PCI}$ group of 60 cases (51 males and 9 females) with mean age $(60.11 \pm 10.96)$ years old, and the other primary PCI group of 60 cases (42 males and 18 females) with mean age $(64.33 \pm 11.91)$ years. The TIMI flow before and after operation were observed in all cases angiographically.

Results By using tirofiban before operation improved TIMI flow, a greater percentage of TIMI 1 grade flow was achieved in the tirofiban + PCI group compared with the primary PCI group before operation (13.3.8\% vs 3.3\%, p>0.05). There was no difference of TIMI 3 grade flow between the two groups after operation, TIMI 2 grade flow was lesser in tirofiban $+\mathrm{PCI}$ group $(6.7 \%$ vs $3.4 \%$, $\mathrm{p}>0.05)$. Reperfusion arrhythmia was lesser in tirofiban $+\mathrm{PCI}$ group (3.4\% vs $6.7 \%$ ).

Conclusion Tirofiban may improve TIMI flow of the IRA in patients with STEMI during emergency PCI.

\section{e0486 TRANSCATHETER CLOSURE OF NO RIM LARGE ATRIAL SEPTAL DEFECT WITH AMPLATZER OCCLUDERS TECHNICAL CONSIDERATIONS, SAFETY AND FEASIBILITY}

doi:10.1136/hrt.2010.208967.486

Chen Lianglong, Luo Yukun, Lin Chaogui, Peng Yafei, Zheng Xingchen. Fujian Union Hospital

Backgrounds Transcatheter closuring no rim or large atrial septal defect (ASD) with Amplatzer occluding device (AOD) was technically challenged. The present study was to address technical issues, and to test the safety and feasibility for transcatheter closuring large and no rim ASDs with AOD.

Methods Patients, with large ASDs of $325 \mathrm{~mm}$ and with no rims at least in one defect border detected echocardiographically, were included in the study. 49 patients eligible underwent transcatheter closure of ASDs and divided into group A ( $n=26$, large ASDs with intact rims) and group $B(n=23$, large ASDs with no rims). Three occluding methods i.e. the conventional releasing, the waist prereleasing, and the dumbbell-shaped releasing were sequentially 